\title{
New Results in Laboratory X-ray Astrophysics
}

\author{
P. Beiersdorfer, H. Chen
}

Lawrence Livermore National Laboratory, Livermore, CA 94550

\author{
K. R. Boyce, G. V. Brown, R. L. Kelley, F. S. Porter, C. K. Stahle \\ NASA Goddard Space Flight Center, Greenbelt, MD 20771
}

J. K. Lepson, J. G. Jernigan

Space Science Laboratory, University of California, Berkeley, CA, 94720

B. J. Wargelin

Harvard-Smithsonian Center for Astrophysics, Cambridge, MA 02138

\author{
S. M. Kahn
}

Physics Department, Stanford University, Stanford, CA 94305

\begin{abstract}
A multi-faceted, multi-institutional laboratory astrophysics program is carried out at the Livermore electron beam ion trap facility, which is a mature spectroscopic source with unsurpassed controls and capabilities, and an unparalleled assortment of spectroscopic equipment, including a full complement of grating and crystal spectrometers and a $6 \times 6$ micro-calorimeter array. Recent results range from the calibration of $x$-ray diagnostics, including the Fe XVII and Fe XXV emission lines, extensive lists of L-shell ions, the first laboratory simulation and fit of a cometary $\mathrm{x}$-ray emission spectrum, and the discovery of new spectral diagnostics for measuring magnetic field strengths.
\end{abstract}

\section{Introduction}

Addressing the atomic needs of the ASCA and EUVE missions, we initiated a laboratory astrophysics program in the the early 1990's. The need for reliable laboratory data continued with the advent of observations with Chandra and XMM-Newton, which provide both CCD-quality and high-resolution grating spectroscopic data. Our laboratory astrophysics program has various facets tailored to address the particular needs for atomic data and calibrated line diagnostics. These include measurements in the extreme ultraviolet and X-ray regions, including measurements of charge exchange, relevant to space astrophysics and planetary science research.

Our program is centered on the electron beam ion traps at the University of California Lawrence Livermore National Laboratory. In over 15 years of use, this facility has been optimized for laboratory astrophysics and provides a unique resource for assessing atomic data and addressing spectroscopic issues. A large suite of spectrometers has been developed, including high-resolution crystal and 
grating spectrometers and a 6x6-pixel, square-array microcalorimeter. We also make use of additional laboratory sources to address a specific astrophysical problem.

A review of laboratory $\mathrm{X}$-ray astrophysics was recently given by Beiersdorfer (2003). Here we only briefly mention a few results and their implications for astrophysics.

\section{Spectral Catalogues}

Line lists covering the X-ray and extreme ultraviolet wavelength bands are still grossly incomplete making it difficult to identify many lines observed with Chan$d r a$ and $X M M$ or to assess line blends appropriately (Lepson et al. 2000; Beiersdorfer et al. 2000b). In response, we have embarked on an ambitious program to provide wavelengths, line identifications, and relative intensities of all relevant lines in the EUV and X-ray region. Recently, we have reported line lists for the L-shell transitions of Ar IX through Ar XVI, Fe VII through Fe IX, and Fe XVIII through Fe XXVI (Brown et al. 2002; Lepson et al. 2002; Lepson et al. 2003).

Our wavelengths are being incorporated in various spectral modeling codes, where they make a decisive difference in line identification and modeling. This was pointed out, for example, by Behar, Cottam, \& Kahn (2001) in their analysis of the L-shell iron emission from Capella observed by Chandra. Our line lists have also resulted in new line identifications, such as that of an Ar IX line near $50 \AA$ in Procyon (Lepson et al. 2003), as well as several revised line assignments.

Our line surveys have uncovered new spectral diagnostics. We recently discovered the first X-ray line that can be used for magnetic field measurements (Beiersdorfer et al. 2003b).

A typical spectrum showing the iron L-shell emission between $15-17 \AA$ is shown in Fig. 1. The spectrum was recorded with our high-resolution grating spectrometer and illustrates the blending of the Fe XVII lines with lines from Fe XVI.

\section{Excitation Cross Sections}

Knowledge of line formation processes and accurate excitation cross sections are needed for modeling observed line intensities and for deriving plasma conditions of the sources under investigation. Focusing on the iron L-shell emission, we have reported detailed excitation cross sections for Fe XXI through Fe XXIV (Gu et al. 1999; Gu et al. 2001; Chen et al. 2002). Our measurements illustrated the importance of resonance excitation and blending with satellite lines produced by dielectronic recombination into high principal quantum numbers. Very importantly, our measurements pointed out inaccuracies in the energy scaling of the excitation rates used, for example, in the MEKAL spectral model. Contributions by dielectronic satellite lines were also studied in the K-shell spectrum of neon and reported recently (Wargelin, Kahn, \& Beiersdorfer 2001). 


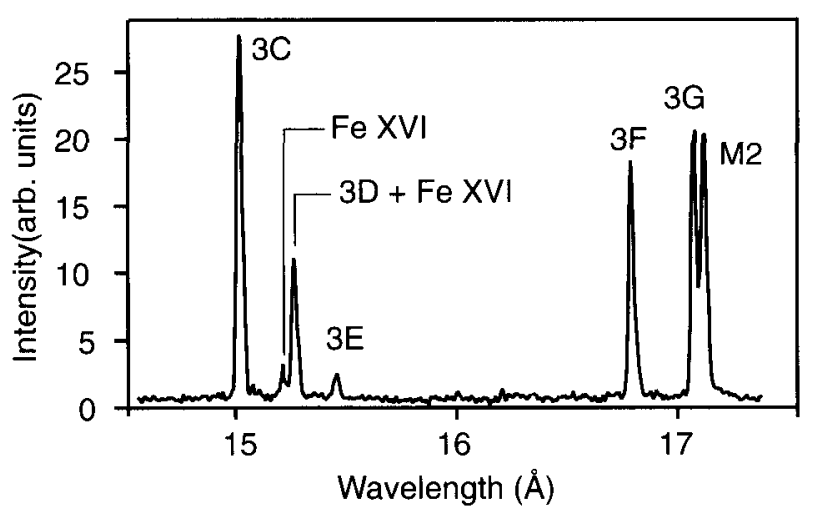

Figure 1. L-shell X-ray emission from iron. The Fe XVII line $3 D$ is blended with a Fe XVI line increasing its apparent intensity relative to that of the $\mathrm{Fe}$ XVII line $3 C$.

\section{The Fe XVII spectrum}

The difficulties of spectral models in predicting reliable line intensities are illustrated by the Fe XVII spectrum. Invariably, the Fe XVII emission could not be described by calculations. The discrepancies between the modeling calculations and the astrophysical spectra have been attributed to various source-specific processes, such as resonant scattering. However, this did not make sense in many instances.

We have studied the L-shell emission of neon-like ions in general and Fe XVII in particular since the beginning of our laboratory astrophysics program (Beiersdorfer et al. 1990; Beiersdorfer \& Wargelin 1994). We showed that the laboratory data reproduce nicely the astrophysical spectra. To do so we used not only the Livermore electron beam ion traps but also tokamak plasmas generated at the Princeton Plasma Physics Laboratory (Brown et al. 1998; Beiersdorfer et al. 2000a; Beiersdorfer et al. 2001; Brown et al. 2001a; Brown et al. 2001b; Brown et al. 2002). Our measurements showed that the discrepancies between models and astrophysical spectra are clearly a problem with the atomic data in all but a few exceptional cases. By contrast, laboratory data obtained with a single-pixel calorimeter by Laming et al. (2000) at the National Institute of Standards and Technology seemed to confirm simple Fe XVII models and to disagree with solar and astrophysical data. We showed these conclusions to be erroneous (Beiersdorfer et al. 2002).

\section{The Fe XXV spectrum}

We have made extensive high-resolution crystal spectrometer measurements of the K-shell iron spectrum in the past (Brown et al. 1989; Beiersdorfer et al. 1992; Wong et al. 1995; Decaux et al. 1995; Beiersdorfer et al. 1996; Decaux et al. 1997; Jacobs, Decaux, \& Beiersdorfer 1997). Most recently, we reported on $\mathrm{K}$-shell line formation by inner-shell ionization (Decaux et al. 2003). We have 


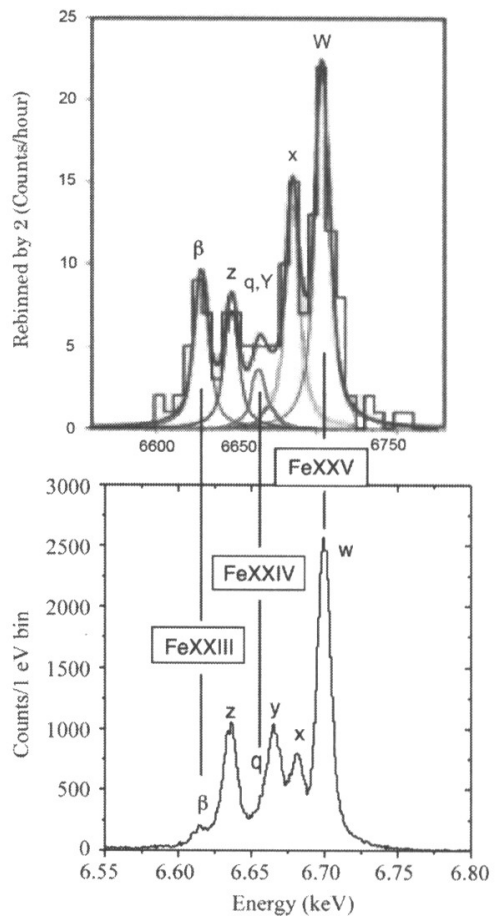

Figure 2. K-shell X-ray emission from iron. Top: Single-pixel calorimeter spectrum obtained at the National Institute of Standards and Technology [adapted from Silver et al. (2002)]. Bottom: Spectrum obtained with the $6 \mathrm{x} 6$ array calorimeter at Livermore. Lines $\beta, q$, and $w$ are used to determine the relative abundances of Fe XXIII, Fe XXIV, and Fe XXV, respectively. 
also used the 36-pixel microcalorimeter to measure the Fe XXV spectrum on one of the Livermore electron beam ion traps (Porter et al. 2000), as the iron K spectrum will be a focus of the upcomming ASTRO-E2 mission. The spectrum is shown in Fig. 2 and compared to the spectrum measured with the single-pixel calorimeter by Silver et al. (2002) at the National Institute of Standards and Technology. ${ }^{8}$

Our spectrum shows the Fe XXV resonance line $w$, inter-combination lines $x$ and $y$, and forbidden line $z$, and the Fe XXIV line $q$ and Fe XXIII line $\beta$. The spectrum matches our crystal spectrometer data as well as the spectra obtained from other high-temperature plasmas such as tokamaks or the Sun (Bitter et al. 1979; Doschek 1990; Kato et al. 1997). The single-pixel calorimeter spectrum, however, whose L-shell branch was used in consecutive measurements to determine Fe XVII ratios mentioned above, differs remarkably from ours. The large intensity of lines $w$ and $\beta$ and small intensity of line $q$ (blended with y) indicates a large abundance of helium-like and beryllium-like iron (Fe XXIII and $\mathrm{Fe} \mathrm{XXV}$ ) and little lithium-like iron (Fe XXIV). This is in contrast to any expected reasonable charge state distribution. Moreover, the $1 s 2 p^{3} P_{2} \rightarrow 1 s^{2}{ }^{1} S_{0}$ inter-combination line $x$ is the second strongest line in this spectrum, although line formation processes do not preferentially favor this transition.

\section{X-rays from Comets}

Our laboratory measurements successfully simulated the X-ray emission spectrum of comet Linear S4 1999 observed with Chandra. We showed that charge exchange between heavy ions found in the solar wind and neutral cometary gases completely reproduces the observed emission (Beiersdorfer et al. 2003a). This laid to rest the question whether the emission is from the solar wind or from the comet itself. The laboratory simulation shows that all X-ray emission stems from the solar wind ions.

Work by the University of California Lawrence Livermore National Laboratory was performed under the auspices of the US Departments of Energy under Contract No. W-7405-Eng-48. Support from NASA's Space Research and Analysis Program and NASA's Planetary Atmospheres Program is gratefully acknowledged.

\section{References}

Behar, E., Cottam, J., \& Kahn, S. M. 2001, Astrophys. J., 548, 966

Beiersdorfer, P. 2003, Ann. Rev. Astron. Astrophys., 412, 343

Beiersdorfer, P., Behar, E., Boyce, K. R., Brown, G. V., Chen, H., Gendreau, K. C., Gu, M.-F., Gygax, J., Kahn, S. M., Kelley, R. L., Porter, F. S., Stahle, C. K., \& Szymkowiak, A. E. 2002, Astrophys. J. (Lett.), 576, L169

Beiersdorfer, P., Boyce, K. R., Brown, G. V., Chen, H., Kahn, S. M., Kelley, R. L., May, M., Olson, R. E., Porter, F. S., Stahle, C. K., \& Tillotson, W. A. 2003a, Science, 300,1461

\footnotetext{
${ }^{8}$ The latter is the same spectrum shown on the poster of the 25th Generally Assembly of the IAU Joint Discussion 17.
} 
Beiersdorfer, P., Brown, G. V., Chen, H., Gu, M.-F., Kahn,S. M., Lepson, J. K., Savin, D. W. \& Utter, S. B. 2000a, in Atomic Data Needs for X-ray Astronomy, ed. M. A. Bautista, T. R. Kallman, and A. K. Pradhan, Vol. NASA/CP-2000-209968, NASA Goddard Space Flight Center (Greenbelt, MD: NASA), 103

Beiersdorfer, P., Brown, G. V., Drake, J. J., Gu, M.-F., Kahn, S. M., Lepson, J. K., Liedahl, D. A., Mauche, C. W., Savin, D. W., Utter, S. B., Wargelin, B. J. 2000b, Revista Mexicana Astronomía y Astrofísica, 9, 123

Beiersdorfer, P., Osterheld, A. L., Chen, M. H., Henderson, J. R., Knapp, D. A., Levine, M. A., Marrs, R. E., Reed, K. J., Schneider, M. B., \& Vogel, D. A. 1990, Phys. Rev. Lett., 65, 1995

Beiersdorfer, P., Phillips, T., Jacobs, V. L., Hill, K. W., Bitter, M., von Goeler, S., \& Kahn, S. M. 1993, Astrophys. J., 409, 846

Beiersdorfer, P., Phillips, T. W., Wong, K. L., Marrs, R. E. \& Vogel, D. A. 1992, Phys. Rev. A, 46, 3812

Beiersdorfer, P., Scofield, J. H., \& Osterheld, A. L. 2003b, Phys. Rev. Lett., 90, 235003

Beiersdorfer, P., Vogel, D. A., Reed, K. J., Decaux, V., Scofield, J., Widmann, K., Hölzer, G., Förster, E., Wherhan, O., Savin, D. W., \& Schweikhard, L. 1996, Phys. Rev. A, 53, 3974

Beiersdorfer, P., von Goeler, S., Bitter, M., \& Thorn, D. B. 2001, Phys. Rev. A, 64, 032705

Beiersdorfer, P. \& Wargelin, B. J. 1994, Rev. Sci. Instrum., 65, 13

Bitter, M., Hill, K. W., Sauthoff, N. R., Efthimion, P. C., Meservey, E., Roney, W., von Goeler, S., Horton, R., Goldman, M., \& Stodiek, W. 1979, Phys. Rev. Lett., 43, 129.

Brown, C. M., Feldman, U. Doschek, G. A., Seely, J. F., LaVilla, R. E., Jacobs, V. L., Henderson, J. R., Knapp, D. A., Marrs, R. E., Beiersdorfer, P. \& Levine, M. A., 1989, Phys. Rev. A, 40, 4089

Brown, G. V., Beiersdorfer, P., Chen, H., Chen, M. H., \& Reed, K. J. 2001a, Astrophys. J., 557, L75

Brown, G. V., Beiersdorfer, P., Kahn, S. M., Liedahl, D. A. \& Widmann, K. 1998, Astrophys. J., 502, 1015

Brown, G. V., Beiersdorfer, P., Liedahl, D. A., Widmann, K., Kahn, S. M., \& Clothiaux, E. J. 2002, Ap. J. Suppl., 140, 589

Brown, G. V., Beiersdorfer, P., \& Widmann, K. 2001b, Phys. Rev. A, 63, 032719

Chen, H., Beiersdorfer, P., Scofield, J. H., Gendreau, K. C., Boyce, K. R., Brown, G. V., Kelley, R. L., Porter, F. S., Stahle, C. K., Szymkowiak, A. E., \& Kahn, S. M. 2002, Astrophys. J. (Lett.), 567, L169

Decaux, V., Beiersdorfer, P., Kahn, S. M., \& Jacobs, V. L. 1997, Astrophys. J., 482, 1076

Decaux, V., Beiersdorfer, P., Osterheld, A., Chen, M., \& Kahn, S. M. 1995, Astrophys. J., 443,464

Decaux, V., Jacobs, V. L., Beiersdorfer, P., Liedahl, D. A., \& Kahn, S. M. 2003, Phys. Rev. A, 68, 012509

Doschek, G. A. 1990, Astroph. J. Suppl., 73, 117

Gu, M.-F., Kahn, S. M., Savin, D. W., Behar, E., Beiersdorfer, P., Brown, G. V., Liedahl, D. A., \& Reed, K. J. 2001, Astrophys. J., 563, 462

Gu, M., Kahn, S., Savin, D., Beiersdorfer, P., Brown, G., Liedahl, D., Reed, K., Bhalla, C., \& Grabbe, S. 1999, Astrophys. J., 518, 1002

Jacobs, V. L., Decaux, V., \& Beiersdorfer, P. 1997, J. Quant. Spectr. Rad. Transf., 58, 645 
Kato, T., Safronova, U. I., Shlyaptseva, A. S., Cornille, M., Dubau, J., \& Nilsen, J. 1997, At. Data Nucl. Data Tables, 67, 225

Laming, J. M., Kink, I., Takacs, E., Porto, J. V., Gillaspy, J. D., Silver, E. H., Schopper, H. W., Bandler, S. R., Brickhouse, N. S., Murray, S. S., Barbera, M., Bhatia, A. K., Doschek, G. A., Madden, N., Landis, D., Beeman, J., \& Haller, E. E. 2000, Astrophys. J., 545, L161

Lepson, J. K., Beiersdorfer, P., Behar, E., \& Kahn, S. M. 2003, Astrophys. J., 590, 604

Lepson, J. K., Beiersdorfer, P., Brown, G. V., Kahn, S. M., Liedahl, D. A., Mauche, C. W., \& Utter, S. B. 2000, RevMexAA, 9, 137

Lepson, J. K., Beiersdorfer, P., Brown, G. V., Liedahl, D. A., Brickhouse, N. S., Dupree, A. K., Kaastra, J. S., Mewe, R., \& Kahn, S. M. 2002, Astrophys. J., 578, 648

Porter, F. S., Audley, M. D., Beiersdorfer, P., Boyce, K. R., Brekosky, R. P., Brown, G. V., Gendreau, K. C., Gygax, J., Kahn, S., Kelley, R. L., Stahle, C. K., \& Szymkowiak, A. E. 2000, Proc. SPIE, 4140, 407

Silver, E., Schnopper, H., Bandler, S., Brickhouse, N., Murray, S., Barbera, M., Takács, E., Gillaspy, J. D., Porto, J. V., Kink, I., Ratliff, P. R., Hudson, L., Laming, J. M., Madden, N., Landis, D., Beeman, J., Haller, E. E., \& Schuch, R. 2002, in Photonic, Electronic \& Atomic Collisions, ed. by J. Burgdörfer and J. S Cohen and S. Datz and C. R. Vane (Rinton Press, Princeton, 2003), p. 312

Wargelin, B. J., Kahn, S. M., \& Beiersdorfer, P. 2001, Phys. Rev. A, 63, 022710

Wong, K. L., Beiersdorfer, P., Reed, K. J., \& Vogel, D. A. 1995, Phys. Rev. A, 51, 1214 